\title{
Procesos de interacción en Instagramers latinoamericanas. El caso de Perú y Colombia durante el 2019
}

\author{
Interaction processes in Latin American Instagramers. The case \\ of Peru and Colombia during 2019
}

\author{
Gonzalez-Carrion, E. L. y Aguaded, I. ${ }^{1}$ \\ Recibido: 15-05-2020 - Aceptado: 10-08-2020 \\ https://doi.org/10.26441/RC19.2-2020-A9
}

RESUMEN: Con el objetivo de evaluar las formas de interacción en las principales Instagramers de Perú y Colombia, se efectuó un análisis exploratorio de sus perfiles en la plataforma audiovisual Instagram durante el año 2019. Por esta razón, primeramente, se recurre a la herramienta Heepsy para establecer las influenciadoras con mayor injerencia dentro de la plataforma en ambos países y posteriormente, a través de las herramientas de monitoreo Fan Page Karma y Social Blade, determinar un conjunto de variables y parámetros respecto a su interacción. Los resultados visibilizan que el índice de audiencia es mayor para las Instagramers de Colombia que para Perú. Además, se observa una clara tendencia por parte de los públicos digitales en valorar el contenido a través de "me gusta" como muestra de una interacción con él, pero en contraparte, se demuestra una disminución representativa en expresar con palabras y en comentarios sus impresiones respecto a lo que observan; esto queda establecido también en los bajos niveles de compromiso que registran la mayoría de Instagramers.

Palabras clave: instagram; redes sociales; participación; comunicación; interacción.

\begin{abstract}
In order to evaluate the forms of interaction in the main Instagramers from Peru and Colombia, an exploratory analysis of their profiles was carried out on the Instagram audiovisual platform during 2019. For this reason, firstly, the Heepsy tool was used to establish the influencers with greater impact within the platform in both countries and then, through the monitoring tools Fan Page Karma and Social Blade, to determine a set of variables and parameters regarding their interaction. The results show that the audience index is higher for Instagramers from Colombia than for the ones from Peru. In addition, there is a clear trend among digital audiences to value content through likes as a sign of interaction with it, but on the other hand, there is a representative decrease in expressing their impressions with words and comments regarding what they observe; this is also established by the low levels of commitment registered by most Instagramers.
\end{abstract}

Keywords: instagram; social net 2works; participation; communication; interaction.

\footnotetext{
${ }^{1}$ Erika-Lucia Gonzalez-Carrion es Máster Interuniversitario en Comunicación y Educación Audiovisual por la Universidad Internacional de Andalucía y la Universidad de Huelva, Doctoranda del Programa Interuniversitario en Comunicación de las universidades de Cádiz, Huelva, Málaga y Sevilla, y Docente de la Universidad Nacional de Loja, Ecuador. erika.gonzalez@unl.edu.ec, https://orcid.org/0000-0003-3808-5460

Ignacio Aguaded es Doctor en Psicopedagogía, Catedrático del Departamento de Educación de la Universidad de Huelva, Presidente del Grupo Comunicar, Director del Máster Internacional Interuniversitario de Comunicación y Educación Audiovisual, y Director del Programa Interuniversitario de Comunicación. Premio de Mejor Investigador de la Universidad de Huelva 2015 en Ciencias Sociales. aguaded@uhu.es, https://orcid.org/0000-0002-0229-1118
} 


\section{Introducción}

El impacto visual es la base para el intercambio comunicativo en Instagram, por medio de imágenes o contenido audiovisual que inducen a que el público espectador realice varias actividades en la Web. Comúnmente, el uso excesivo de texto no es una de las características principales de las publicaciones que aquí acontecen, donde también el usuario tiene la potestad de elección tanto de contenido, como del dispositivo desde el que quiere realizar su navegación.

Como bien lo destaca Padrini (2015), la acción de fotografiar que facilita Instagram, simboliza también un contacto y una relación con el entorno que rodea al usuario, permitiendo expandirse a otros espacios cercanos o ajenos. En este marco, Gómez (2012) sostiene que la fotografía es clave para la generación de conexiones sociales y a nivel de plataformas digitales, se busca cambiar el rol tradicional de la imagen hasta el punto de convertirla en una representación.

Tanto en los perfiles comerciales o empresariales, Instagram fomenta un diálogo con la tecnología como mediadora, con la posibilidad de reacciones inmediatas y donde la diversificación del contenido que se propone alcanza límites notables. En el mismo espacio convergen tanto los creadores de material audiovisual como quienes lo reciben, convirtiéndose incluso a posterior en prosumidores de esta información. Es así que autores como Matley (2018, p. 67), aseguran que Instagram "proporciona ventajas clave para la promoción estratégica y el marketing de cada persona, como filtros que permiten a los usuarios modificar fotos, dándoles un mayor control sobre la imagen final".

Además, Instagram tiene características que pueden contribuir o inhibir la exposición y evitación selectiva, es decir, no requiere reciprocidad para que los usuarios sigan determinado perfil (Parmeleee y Roman, 2020). Esta plataforma no es simplemente un álbum de fotos en línea, sino un vehículo para la conexión social, la expresión y la comunicación íntima a través de imágenes (Lowe-Calverley, Grieve y Padgett, 2020).

Se forma así un vínculo inmediato de comunicación, dentro de una plataforma que se rige por una estética muy particular que transmite contenido de forma diferente. En opinión de Fernández (2016), surgen dos conceptos que condicionan estas dinámicas; el engagement que constituye la interactividad suscitada dentro de la plataforma y aquellos conceptos referidos a las colaboraciones y participaciones en cada uno de los posteos que se emiten.

Como plantea Kim y Kim (2019), Instagram se consolida como el sitio de redes sociales fotocéntrico más utilizado y, los usuarios cargan una variedad de fotos que manifiestan historias sobre sí mismos y el mundo que ven. Ichau, Frissen y d'Haenens (2019) sugieren que Instagram utiliza un léxico específico que lo conecta con las audiencias, promoviendo una fácil interpretación. Conjuntamente, y según destaca Couture (2020), Instagram incluso facilita la edición digital de las de fotografías antes de ser publicadas, centrándose en lo visual como punto clave para generar una participación de los usuarios.

En virtud de estas funcionalidades y de forma similar a Google, Instagram empieza a conceptualizarse como un buscador muy productivo de material netamente audiovisual, donde el usuario puede acceder a galerías ilimitadas y con una calidad bastante óptima. Para Tiggemann y Barbato (2018), Instagram alienta a los usuarios a hacer comentarios sobre las fotos de otros, los mismos que son expuestos para que otros los vean. Las diferentes formas de participación que acontecen en este medio se nutren de todas las factibilidades que como red digital tiene Instagram y buscan por medio de ellas generar una reacción en los usuarios.

Instagram también fomenta el intercambio activo y las redes sociales, de tal forma que los usuarios pueden compartir una imagen en otros sitios de redes sociales, al mismo tiempo que publican (Lee y Chau, 2018). Son precisamente las imágenes que aparecen en este entorno, un reflejo a través 
del cual los usuarios transmiten autenticidad y su recuerdo más preciado para obtener un feedback representativo (Colliander y Marder, 2018).

Autores como Longobardi, Settani, Fabris y Marengo (2020, p. 1) consideran que los "dos indicadores que proporcionan información sobre la posición del usuario entre la comunidad de Instagram son: el número de seguidores de Instagram obtenidos y, el número de otros usuarios de Instagram a los que el usuario se ha suscrito". Venus y Ryu (2020) manifiestan que quienes obtienen mayor notoriedad en estos rangos, son los influenciadores, celebridades digitales que publican una amplia variedad de fotos (selfies atractivas, fotos grupales, retratos glamorosos tomados por otros, fotos de productos o marcas, viajes u otros), exhibiendo estratégicamente una gama de fotos para promocionar su estilo de vida o la marca que representan.

Según Casaló, Flavián e Ibáñez-Sánchez (2018), estos referentes digitales construyen sus perfiles de liderazgo, porque Instagram, debido a su naturaleza inherentemente visual, brinda una gran oportunidad para difundir nuevas tendencias. La estructura de plataformas como Instagram, facilita a criterio de Rietveld, Van Dolen, Mazloom y Worring (2020), la creación medios propios, cuyo objetivo final es relacionarse con los seguidores mediante la construcción de publicaciones y por medio de modalidades visuales y textuales dentro de los contenidos.

Los influenciadores como personas de referencia en panoramas 2.0, se benefician de una digitalización y transmisión de constante información por nuevos canales, mejorando y expandiendo su comunicación, en varias direcciones, en un mundo actual y cambiante (Stevenson, Oluranti, Edemode y Anyanwu, 2018). Sustentando este criterio, Huang y Su (2018), expresan que el trabajo de Instagram enfocado en resaltar el aspecto visual como forma de comunicación, es plenamente aceptado y responde a las necesidades actuales de cada persona.

Aunque Instagram se convierte en uno de los atractivos más fuertes para las generaciones más jóvenes, Larsson (2017) acota que, también es necesario resaltar las implicaciones que tiene la plataforma en los medios de comunicación y las tendencias que marca respecto a cómo se desarrolla la interacción. Para Riquelme (2017), se instaura una idea de que aplicaciones como Instagram generan un nivel de eficacia dadas sus características vinculadas al feedback que se produce y a la velocidad de participación que mantienen.

Los influenciadores que aquí se desempeñan se valen de elementos creados específicamente para incitar una reacción. Así, plataformas como Instagram permiten emitir comentarios, crear frases virales en cuestión de segundos, insertar elementos como los conocidos emojis, una simbología surgida en la red y otros aditamentos que proponen un modelo comunicacional con recursos diferentes, innovadores. Pareciera a simple vista que la función de estos elementos es acompañar el material audiovisual que se expone, pero su implicación va mucho más allá, buscan despertar una emoción y posterior acción concreta en los usuarios, sea para fines sociales o económicos.

Diferentes Instagramers comparten sus opiniones, puntos de vista, experiencias e incluso actividades rutinarias diarias con fuerte influencia en sus audiencias, fanáticos y seguidores en todo el mundo; se genera una combinación de recursos audiovisuales para la difusión de contenido y un mayor alcance (Arora, Bansal, Kandpal, Aswani y Dwivedi, 2019). En esta línea, Jackson y Luchner (2018) plantean que la personalidad de un individuo en estos contextos digitales influye en cómo se presenta en Instagram y afecta su respuesta emocional a las respuestas que recibe en la plataforma.

En virtud de esto, la emisión y recepción de contenidos en Instagram no se limita únicamente a la relación entre usuarios y productores de material, abarca también áreas como la publicitaria o promocional, claves en el desarrollo de una identidad digital y más para aquellos que se convierten en 
referentes o influenciadores dentro de Instagram. Despard (2015: p. 4) considera que: "Esta vaguedad en relación con el contenido corresponde a una subestimación de las diversas formas en que se utilizan las cuentas de Instagram: por ejemplo, no solo como una forma de comunicarse con amigos, sino también como herramientas de marketing, publicidad y sensibilización, o como galerías, portafolios o revistas visuales para profesionales creativos".

El contenido dinámico de Instagram, según Wilkinson (2018), presenta diversas oportunidades para el disfrute de sus consumidores, que encuentran en este conjunto de información, miradas y perspectivas diferentes sobre un particular que resulte de interés para el usuario, tanto por cómo están constituidos los materiales audiovisuales en la plataforma, como por la idea que transmiten sin necesidad de los objetos o elementos que integran. Como manifiestan Baulch y Pramiyanti (2018), la relevancia de Instagram en la cultura audiovisual actual es de tal dimensión que la comunicación ha pasado de un aspecto tradicional a otro meramente visual.

El desafío actual para quienes se desenvuelven en una plataforma digital como Instagram, es presentar una originalidad con valor agregado en cada una de sus publicaciones, con la suficiente innovación para establecer una ventaja frente a otros medios de naturaleza similar. La participación dentro un medio digital no siempre ocurre de forma fluida, es necesario una organización de los elementos que componen cada posteo y la correcta organización de los recursos a disposición, de modo que todo este conglomerado atraiga a un público hacia un contexto de participación constante donde intervenga directamente de forma natural. Este proceso, tal como refiere Mukherjee y Jansen (2020), se conoce como la segunda pantalla o fenómeno de pantalla cruzada, situación que es visible en plataformas sociales y digitales como Instagram.

Cada medio social tiene un tipo de actores diferentes, que muestra un contacto e interacción, pero con comportamientos que varían de acuerdo con la cultura donde pertenece, su situación geográfica o incluso profesional, lo que resulta en que cada usuario ejerce un poder de influencia y participación que difiere de otro (Asim, Malik, Raza y Shahid, 2019). Las personas influyentes en redes sociales conjugan una serie de estrategias para continuar y expandir de ser posible su estatus de celebridades digitales (Hurley, 2019).

Los influenciadores en redes, principalmente en Instagram, son conscientes que su influencia será determinada por la interacción que consigan con la audiencia a la que dirigen sus publicaciones. Un posteo que no registre participación del público, no puede considerarse un contenido con el poder suficiente para ser considerado influyente; surge la necesidad inmediata de aprender y poner en ejercicio todo código digital que conecte y principalmente que muestre un lenguaje universal, entendible para todos.

No obstante, y según lo manifiestan Nandagiri y Philip (2018), algunos influenciadores aún no obtienen un reconocimiento social por el trabajo que realizan, pese a esto, con el auge de redes sociales, muchas empresas se están dando cuenta del potencial que poseen. Las potencialidades del influenciador respecto a su poder comunicacional, son variadas y según indican Martínez-Sanz y González-Fernández (2018), van "desde mensajes puramente informativos, entre los que se incluyen las recomendaciones o consejos prácticos, al relato de experiencias, formas de vida u opiniones" (p.236). Estos contenidos, a juicio de Weismueller, Harrigan, Wang y Soutar (2020), desembocan en que las personas o consumidores interesados en un área en particular sigan e interactúen con personas influyentes para mantenerse al día con las últimas noticias y tendencias.

Respondiendo a estos antecedentes, la presente investigación busca determinar mediante un conjunto de variables establecidas, los índices de interacción en las principales Instagramers de Perú y Colombia, para establecer cómo se desarrolla el intercambio comunicativo con sus seguidores. 


\section{Metodología}

La presente investigación emplea una metodología cuantitativa a través de la descripción de datos. Se combinan dos herramientas Fan Page Karma y Social Blade para evaluar las principales variables de interacción en los perfiles seleccionados; con la primera herramienta se determina: seguidores, número de me gusta, número de publicaciones, número total de comentarios, total de reacciones, publicaciones al día, comentarios reales por publicación, juntamente con las 10 publicaciones que más alcance tuvieron y el índice por el que lograron esta categorización. Con la herramienta Social Blade se expone: nivel del perfil, el rango de compromiso, el rango en medio y el rango de acuerdo con la cantidad de audiencia que acumula.

La elección de todo el año 2019 como tiempo de análisis se justifica en la necesidad de evaluar un año corrido, con todos los cambios que implica, ya sea por movimiento de audiencia o eventos coyunturales que puedan marcar modificaciones en el comportamiento de la audiencia digital.

\subsection{Muestra}

Para delimitar la muestra, se recurrió a la herramienta de categorización de influenciadores Heepsy, filtrando el rubro de belleza y nuevas tendencias. Con esta información y teniendo en cuenta que al momento no existe una plataforma que identifique a influenciadoras que sean nativos digitales (los listados incluye a figuras de la televisión, músicos, deportistas y más), se procedió a seleccionar entre la muestra arrojada por Heepsy a aquellos perfiles de Instagramers que cumplan las características mencionas por Lario (2019), quien señala a los influenciadores como personas creadoras de contenido digital, que con sus ideas buscan un intercambio y persuasión en quienes los siguen. Bajo este concepto y teniendo en cuenta el rubro seleccionado, se eligió a las cinco Instagramers de Perú y Colombia que producen contenido digital, más allá presentar meramente fotos de sus vidas personales, sino actualizaciones continuas de material audiovisual. En la tabla 1 figuran las instagramers seleccionadas.

Tabla 1. Muestra de investigación

\begin{tabular}{|l|l|}
\multicolumn{1}{|c|}{ Perú } & \multicolumn{1}{c|}{ Colombia } \\
\hline Flavia Laos & Paula Galindo \\
\hline Rosangela Eslo & Daniela Ospina \\
\hline Luciana Fusterg & Amadorat \\
\hline Ximena Hoyos & Matu Garcés \\
\hline Spheffany Loza & Cami Luzuriaga \\
\hline
\end{tabular}

Fuente: Heepsy (2020). Elaboración propia.

En primera instancia se extrajo los datos provenientes de la herramienta Fan Page Karma para posteriormente hacerlo en Social Blade, aprovechando los parámetros que ambas permiten identificar respecto a la interacción de los perfiles y cómo esto se representa en su compromiso, aclarando así su nivel de realidad y certeza.

\section{Resultados}

Los resultados obtenidos permiten una visión más clara sobre la interacción efectuada durante un año completo (2019) en las principales Instagramers de Perú y Colombia, respecto al contenido que proponen y la reacción que obtienen de sus audiencias. Conjuntamente se relaciona los datos encontrados con el nivel de compromiso adquirido por las influenciadoras en cuanto a una comunicación bidireccional con sus audiencias. 
Tabla 2. Interacción de las Instagramers peruanas durante el 2019

\begin{tabular}{|l|c|c|c|c|c|c|c|}
\multicolumn{1}{|c|}{ Instagramer } & Seguidores & $\begin{array}{c}\text { Número de } \\
\text { me gusta }\end{array}$ & $\begin{array}{c}\text { Número de } \\
\text { publicaciones }\end{array}$ & $\begin{array}{c}\text { Número } \\
\text { total de } \\
\text { coment. }\end{array}$ & $\begin{array}{c}\text { Total de } \\
\text { Reacciones }\end{array}$ & $\begin{array}{c}\text { Publicaciones } \\
\text { al día }\end{array}$ & $\begin{array}{c}\text { Comentarios } \\
\text { reales por } \\
\text { publicación }\end{array}$ \\
\hline Flavia Laos & 3.675 .376 & 27.226 .208 & 381 & 180.798 & 27.407 .006 & 1 & 475 \\
\hline Rosangela Eslo & 3.490 .721 & 16.028 .227 & 345 & 229.604 & 16.257 .831 & 0.9 & 665 \\
\hline Luciana Fusterg & 3.412 .661 & 19.244 .958 & 202 & 160.421 & 19.405 .379 & 0.6 & 794 \\
\hline Ximena Hoyos & 1.632 .529 & 5.008 .323 & 172 & 22.317 & 5.030 .640 & 0.5 & 130 \\
\hline Spheffany Loza & 1.425 .380 & 4.017 .483 & 121 & 38.990 & 4.056 .473 & 0.3 & 322 \\
\hline
\end{tabular}

Fuente: Fan Page Karma (2020). Elaboración propia.

Como se muestra en la Tabla 2, las cinco Instagramers en el rubro de belleza y nuevas tendencias acumulan millones de seguidores hasta el 31 de diciembre de 2019, liderando y asemejándose en sus índices tres de ellas: Flavia Laos, Rosangela Eslo y Luciana Fuster, que superan los 3 millones Ximena Hoyos y Spheffany Loza sobrepasan el millón de seguidores. A partir de este punto se empieza a vislumbrar las variables referentes a la interactividad con sus audiencias dentro de la plataforma audiovisual, de esta forma, en números de "me gusta", las Instagramers con mayor cantidad de audiencia, son también las que mayor alcance acaparan, con más de 27 millones para Flavia Laos, más de 16 millones para Ronsangela Eslo y superando los 19 millones Luciana Fuster.

De igual forma, son estas tres influenciadoras las que mayor número de publicaciones efectúan, con 381,345 y 202, manifestando una relación directa entre la producción de contenido digital a través de posteos y los "me gusta" que logran en el público. No obstante, a Ximena Hoyos, que se encuentra en cuarto lugar, solamente la separan 30 publicaciones de su antecesora, por lo que es necesario considerar también el trabajo dentro de las publicaciones y valor diferencial que se aporta para que una supere a otra con millones de "me gusta".

El número de comentarios también asigna liderazgo a las tres Instagramers que vienen como punteras y cuyos valores están por sobre los 180.000 para Flavia Laos, 229.000 para Rosangela Eslo y 160.000 para Luciana Fuster. Existe una diferencia bastante representativa con los otros dos perfiles, más de 100,000 comentarios las separan a las primeras tres. Ahora bien, al analizar el total de reacciones asignadas por la audiencia en cada perfil (que incluyen los emojis que se despliegan al abrir la barra de comentarios en un posteo y significa un esfuerzo más del usuario por participar frente al contenido), las diferencias son mínimas con el número de "me gusta", esto permite deducir que el público casi en su totalidad participa a través de un clic en el corazón que aparece debajo de cada material. La relación se muestra a continuación, observando que la diferencia prácticamente es inexistente.

Gráfico 1. Relación entre "me gusta" y total de reacciones - Instagramers Perú

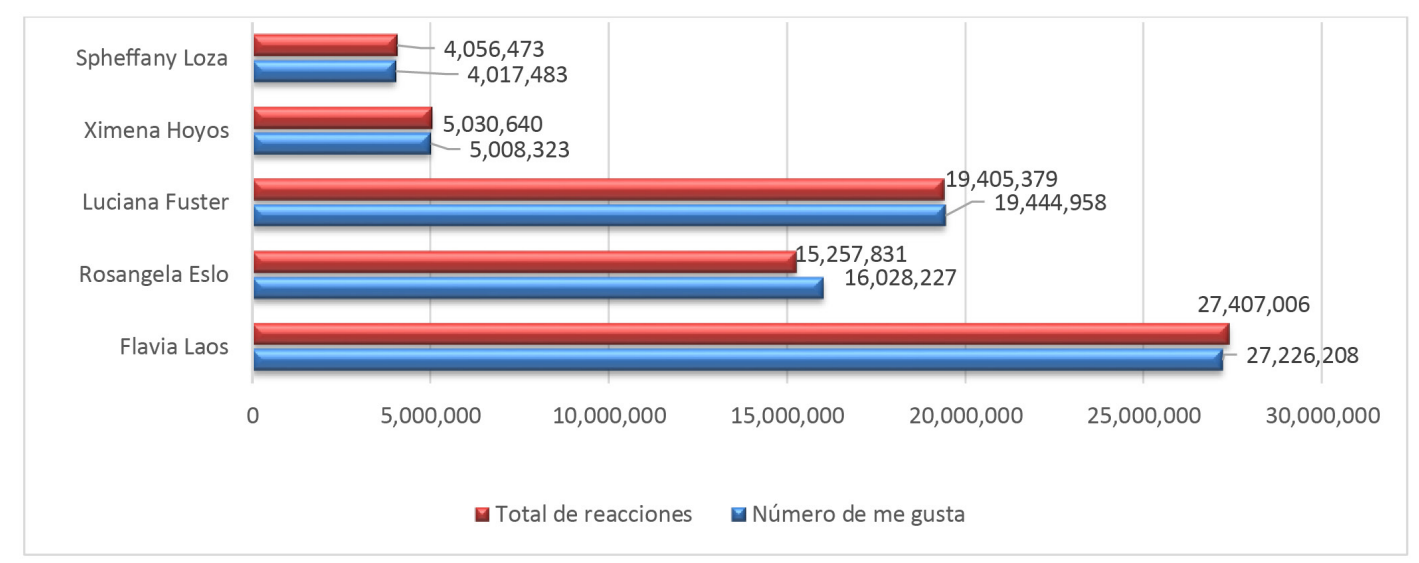

Fuente: Fan Page Karma (2020). Elaboración propia. 
En cuanto a las publicaciones que emiten las Instagramers, la tabla 2 demuestra que el tope máximo es uno por día, lo que evidencia que, si bien el contenido se actualiza, existe una mesura en torno a la estrategia que se utiliza para captar la atención de la audiencia, cuidando de no saturar a un público digital que se enfrenta a una variedad de contenido de magnas proporciones y del cual obtener un "me gusta" u otra reacción no es tarea sencilla. En este sentido, es valorable la filtración de comentarios reales por publicación que permite la herramienta Fan Page Karma, ubicando en primer lugar a Luciana Fuster con un estimado de 794 comentarios por publicación, seguida de Rosangela Eslo con 665 y finalmente, Flavia Laos con 475.

Quienes lideran en el rubro de belleza y nuevas tendencias, presentan una congruencia en las variables de interacción, es decir, no lideran únicamente por la cantidad de seguidores que obtienen, sino por lo que estos ejecutan en sus perfiles y desde sus respectivas cuentas. Al medir la interacción en Instagram en las variables indicadas, se prueba que las cuentas analizadas son capaces de mover comunidades, lograr índices y movimiento dentro de la plataforma, más aún con la capacidad de establecer participaciones reales, que permiten establecer diferencias y marcar liderazgos.

Tabla 3. Interacción de las Instagramers colombianas durante el 2019

\begin{tabular}{|l|r|r|r|r|r|r|r|}
\hline Instagramer & Seguidores & $\begin{array}{c}\text { Número de } \\
\text { me gusta }\end{array}$ & $\begin{array}{c}\text { Número de } \\
\text { publicaciones }\end{array}$ & $\begin{array}{c}\text { Número } \\
\text { total de } \\
\text { coment. }\end{array}$ & $\begin{array}{c}\text { Total de } \\
\text { Reacciones }\end{array}$ & $\begin{array}{c}\text { Publicaciones } \\
\text { al día }\end{array}$ & $\begin{array}{c}\text { Comentarios } \\
\text { reales por } \\
\text { publicación }\end{array}$ \\
\hline Paula Galindo & 8.486 .816 & 66.535 .503 & 233 & 259.691 & 66.795 .194 & 0.6 & 1.114 \\
\hline Daniela Ospina & 6.688 .476 & 25.628 .766 & 244 & 153.852 & 25.782 .618 & 0.7 & 631 \\
\hline Amadorat & 2.815 .771 & 26.377 .249 & 152 & 109.151 & 26.486 .408 & 0.4 & 718 \\
\hline Matu Garcés & 1.595 .009 & 16.301 .188 & 204 & 100.261 & 16.401 .449 & 0.6 & 491 \\
\hline Cami Zuluaga & 1.359 .382 & 5.619 .789 & 86 & 14.877 & 5.634 .665 & 0.2 & 173 \\
\hline
\end{tabular}

Fuente: Fan Page Karma (2020). Elaboración: Propia.

Como se observa en la Tabla 3, en el caso de Colombia las influenciadoras acumulan mayor cantidad de seguidores hasta el 31 de diciembre de 2019, que los perfiles en Perú. Paula Galindo y Daniela Ospina lideran ampliamente en comparación al resto, superando los 8 y 6 millones de seguidores respectivamente; los perfiles restantes oscilan entre 1 y casi 3 millones de seguidores en sus cuentas verificadas. Respecto a la interacción que evidencian, se denota un aumento considerable respecto a Perú, por ejemplo, siendo notable el liderazgo de Paula Galindo con más de 66 millones de "me gusta"; Daniela Ospina y Amadorat abarcan durante el año un índice similar, con 25 y 26 millones de "me gusta" cada una; Matu Garcés y Cami Zuluaga superan los 16 y 5 millones. Sin embargo, la distancia entre la primera Instagramer y el resto dista en gran medida, con una diferencia de 40 millones de "me gusta" respecto quien ocupa el segundo puesto.

Dos de las tres personas que encabezan el listado, también producen la mayor cantidad de publicaciones durante 12 meses, con la particularidad que la Instagram Matu Garcés (una de las que menos cantidad de seguidores tiene), se ubica en tercer lugar con 204 posteos y acortando su diferencia con Paula Galindo y Daniela Ospina. Esto prueba que no existe una relación estrecha entre la cantidad de material subido y el número de adeptos que ganan.

En el número total de comentarios durante el año, nuevamente se impone el liderazgo de Paula Galindo, quien se aproxima a los 260.000 y marca una distancia significativa con las influenciadoras que vienen luego. Daniela Ospina se acerca a los 154.000 comentarios anuales, mientras que Amadorat y Matu Garcés presentan índices relativamente semejantes, sobrepasando los 109.000 y 100.000 respectivamente. Cuando se indaga en el total de reacciones, sucede la misma situación que en Perú, éstas se enfocan en los "me gusta" antes que en la utilización de emojis como reacciones dentro de los comentarios, presentando unas variaciones prácticamente nulas. 
Gráfico 2. Relación entre "me gusta" y total de reacciones - Instagramers Colombia

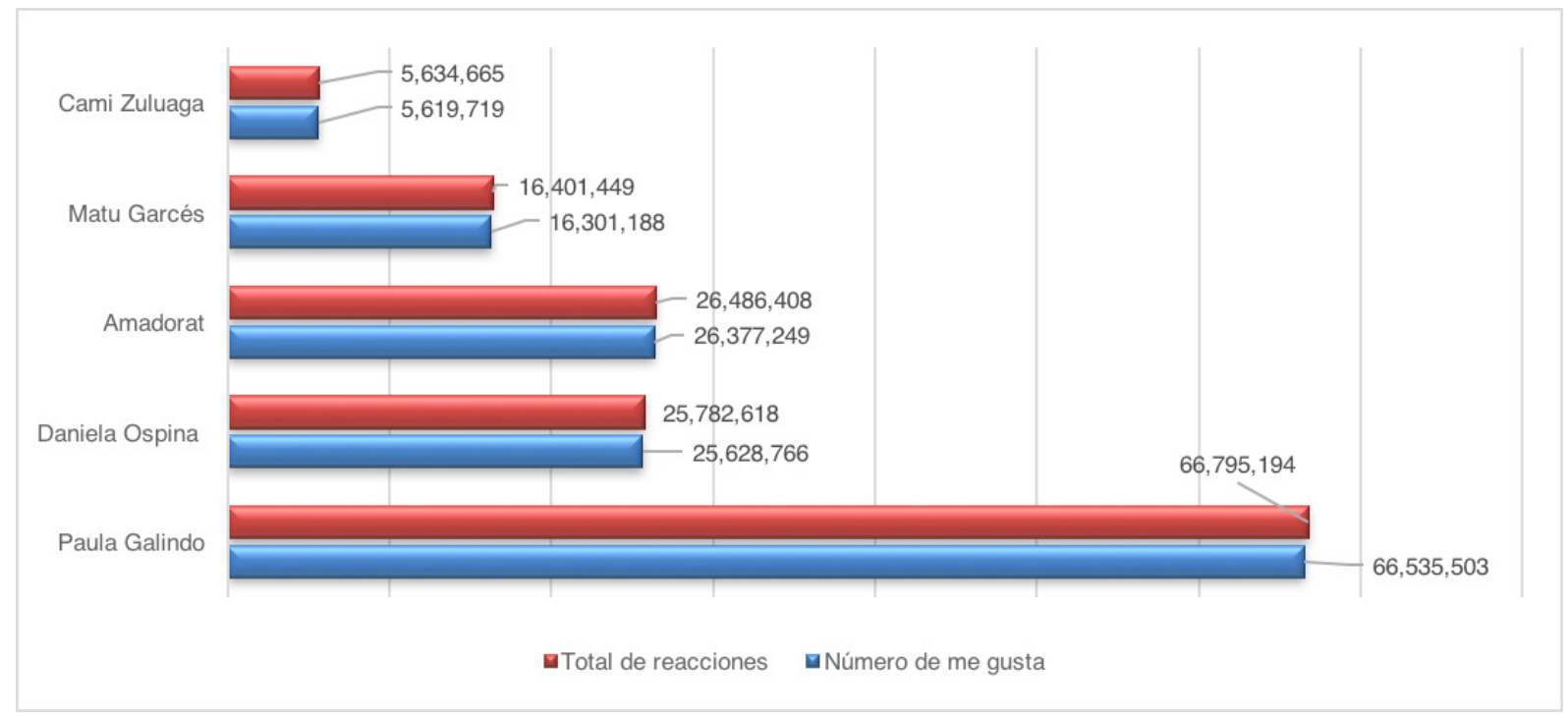

Fuente: Fan Page Karma (2020). Elaboración: Propia.

En el Gráfico 2 se aprecia los comentarios reales en promedio que se recogen por cada publicación y muestran nuevamente que la interacción más elevada de los usuarios es con Paula Galindo con más de mil comentarios en estimación a sus posteos; de cerca la siguen Amadorat y Daniela Ospina con 718 y 631, con un promedio de publicaciones que va de 0.2 a 0.7 la máxima demostrando que no existe una continuidad. Las Instagramers con menor cantidad de comentarios por publicación son Matu Garcés con 491 y Cami Zuluaga con 173, ubicándose así entre las referentes que menor cantidad de interactividad registran con sus audiencias.

Aunque existen algunas diferencias entre Perú y Colombia, se manifiestan semejanzas en cuanto a determinados parámetros de interacción, que se asocian más a la calidad que a la cantidad de estos dentro de Instagram. Infiere, indudablemente, las temáticas que se abarcan y desde qué perspectiva se estructura o configura el contenido que se presentan, pues la participación e interacción de una audiencia, se condiciona a si lo que ve le interesa y puede dedicarle un tiempo. Sabemos que la acción inmediata en Instagram es dar un "me gusta" como símbolo de actuar con el contenido, y por los resultados aún existe una reticencia a aportar mayor cantidad de tiempo a nivel comentarios.

Tabla 4. Categorización, compromiso y rango - Instagramers Perú

\begin{tabular}{|l|c|c|c|c|c|}
\multicolumn{1}{|c|}{ Instagramer } & Grado & $\begin{array}{c}\text { Tasa de } \\
\text { Compromiso }\end{array}$ & $\begin{array}{c}\text { Rango de } \\
\text { Compromiso }\end{array}$ & $\begin{array}{c}\text { Rango en } \\
\text { Medios }\end{array}$ & $\begin{array}{c}\text { Rango respecto } \\
\text { a seguidores }\end{array}$ \\
\hline Flavia Laos & A- & $1.56 \%$ & 6.761 .537 & 800.654 & 3.282 \\
\hline Rosangela Eslo & A- & $0.90 \%$ & 7.796 .697 & 1.935 .916 & 3.590 \\
\hline Luciana Fusterg & A- & $3.76 \%$ & 4.394 .184 & 1.367 .741 & 3.718 \\
\hline Ximena Hoyos & B+ & $2.24 \%$ & 5.906 .818 & 2.972 .684 & 10.738 \\
\hline Spheffany Loza & B+ & $1.50 \%$ & 6.889 .233 & 1.930 .389 & 12.920 \\
\hline
\end{tabular}

Fuente: Social Blade (2020). Elaboración propia.

Ahora, la herramienta de medición Social Blade, permite establecer el grado del canal en función de la interactividad que registra, tal como se expone en la Tabla 4. Es así que en Perú, Flavia Laos, Rosangela Eslo y Luciana Fuster presentan un grado A-, es decir, la actividad que registran está a solo un nivel de lograr el A+, que ubica al perfil como totalmente efectivo dentro de la plataforma; 
las cuentas pertenecientes a Ximena Hoyos y Spheffany Loza registran un B+, que dentro de Social Blade corresponde a las cuentas que vienen realizando un trabajo efectivo, pero aún deben mejorar sus estrategias de comunicación con los internautas.

Respecto a la tasa de compromiso, se observa que el mejor porcentaje lo obtiene Luciana Fuster con $3.76 \%$, a pesar de ubicarse en el tercer lugar en cuento a seguidores; le sigue Ximena Hoyos con $2.24 \%$ ocupando el cuarto lugar y, las demás oscilan entre el $0.90 \%$ y $1.56 \%$ de compromiso. Estos porcentajes demuestran que del número de seguidores no depende la fidelización de la audiencia, en cuanto a fijar un compromiso recíproco entre la audiencia y sus referentes, o viceversa.

A nivel global, como se puede apreciar, tanto en rango de compromiso como rango en medios, las posiciones ocupadas por las Instagramers peruanas son muy lejanas, pero dentro de esto, el mejor posicionamiento lo ocupa es Luciana Fuster, coincidiendo con el nivel de compromiso (interacción) más próspero. No obstante, al visualizar el rango global respecto a seguidores, las posiciones se mantienen.

Tabla 5. Categorización, compromiso y rango - Instagramers Colombia

\begin{tabular}{|l|c|c|r|r|r|}
\multicolumn{1}{|c|}{ Instagramer } & Grado & $\begin{array}{c}\text { Tasa de } \\
\text { Compromiso }\end{array}$ & $\begin{array}{c}\text { Rango de } \\
\text { Compromiso }\end{array}$ & $\begin{array}{c}\text { Rango en } \\
\text { Medios }\end{array}$ & $\begin{array}{c}\text { Rango } \\
\text { respecto a } \\
\text { seguidores }\end{array}$ \\
\hline Paula Galindo & A & $1.64 \%$ & 6.680 .163 & 332.653 & 962 \\
\hline Daniela Ospina & A- & $0.83 \%$ & 7.940 .136 & 623.284 & 1.378 \\
\hline Amadorat & A- & $13.40 \%$ & 920.550 & 2.281 .804 & 4.918 \\
\hline Matu Garcés & B+ & $3.45 \%$ & 4.735 .019 & 1.294 .904 & 11.071 \\
\hline Cami Zuluaga & B+ & $3.77 \%$ & 4.332 .841 & 1.045 .659 & 13.747 \\
\hline
\end{tabular}

Fuente: Social Blade (2020). Elaboración propia.

En la Tabla 5 se vislumbra el caso de Colombia. Paula Galindo presenta un grado A en su cuenta, posicionada por la herramienta Social Blade como efectiva y de injerencia dentro de la plataforma audiovisual. Fuera de ella, los porcentajes de compromiso se presentan la siguiente categorización: Daniela Ospina y Amadorat ostentan un A-, perfiles que, de acuerdo al recurso utilizado, les falta un solo nivel para encontrar una gestión y respuesta en concordancia con los seguidores que acarrea; finalmente Matu Garcés y Cami Zuluaga con un B+ demuestran aún la necesidad de implementar dentro de sus perfiles una comunicación bidireccional capaz de generar un feedback y el que sus audiencias se comprometan (se mantengan atentos a su contenido y no dejen de seguirlas).

Aunque los porcentajes de compromiso no evidencian mayores diferencias con las cuentas de referencia en Perú, se suscita el hecho de que Amadorat eleva a tal nivel su compromiso, que se convierte en la referente con el mejor porcentaje no solo en su país, sino al compararla con la muestra total de análisis (ambos países), llegando a un 13.40\%; este porcentaje ubica a su perfil como el de mejor proyección a futuro, pues es tal su nivel de interactividad que la adopción de nuevos seguidores es cuestión de tiempo para su cuenta.

De forma similar a Perú, en el rango global de compromiso la mejor ubicación la consigue Amadorat que muestra el mejor compromiso, mientras que en rango en medios y respecto al número de seguidores que acumulan, Paula Galindo destaca por su influencia e intercambio comunicativo con su audiencia, lo que repercute en la posición que ocupa y que es destacable teniendo en cuenta el nivel de competitividad existe en la red, precisamente en el rubro estudiado. 
Tabla 6. Publicaciones con mejor repercusión - Instagramers Perú

\begin{tabular}{|l|c|c|c|c|c|}
\multicolumn{1}{|c|}{ Instagramer } & Fecha & Hora & Tema & Me Gusta & Comentarios \\
\hline Flavia Laos & $1 / 06 / 19$ & $15: 23$ & Coreografía & $79 \mathrm{~K}$. & 875 \\
\hline Spheffany Loza & $1 / 06 / 19$ & $12: 41$ & Sorteo & $4.8 \mathrm{~K}$. & 302 \\
\hline Rosangela & $1 / 06 / 19$ & $11: 21$ & Inicio de Mes & $48 \mathrm{~K}$. & 686 \\
\hline Rosangela & $31 / 05 / 19$ & $22: 12$ & 1 er millón TikToK & $38 \mathrm{~K}$. & 994 \\
\hline Luciana Fusterg & $31 / 05 / 19$ & $19: 23$ & Coreografía & $123 \mathrm{~K}$. & $1.2 \mathrm{~K}$. \\
\hline Rosangela & $31 / 05 / 19$ & $19: 16$ & Época invernal & $10 \mathrm{~K}$. & 173 \\
\hline Spheffany Loza & $31 / 05 / 19$ & $18: 09$ & Caos y sencillez & $39 \mathrm{~K}$. & 317 \\
\hline Flavia Laos & $31 / 05 / 19$ & $15: 03$ & Nuevo Proyecto & $31 \mathrm{~K}$. & 283 \\
\hline Rosangela & $30 / 05 / 19$ & $23: 30$ & Valora, agradece & $37 \mathrm{~K}$. & 302 \\
\hline Rosangela & $30 / 05 / 19$ & $19: 34$ & Fin de mes & $37 \mathrm{~K}$. & 289 \\
\hline
\end{tabular}

Fuente: Fan Page Karma (2020). Elaboración propia.

Como se aprecia en la Tabla 6, las 10 publicaciones con mejor repercusión corresponden a cuatro perfiles (ningún posteo de Ximena Hoyos se encuentra dentro del listado). De estas, siete corresponden al mes de mayo, mientras tres a junio de 2019; en su mayoría los posteos se realizan en la tarde y noche de los días indicados (jueves 2 publicaciones, viernes 5 publicaciones y sábado 3 publicaciones), posiblemente por el movimiento e interacción que generan dentro de la plataforma. El número de "me gusta" y "comentarios" que reciben estas publicaciones es proporcional al índice de seguidores con el que cuentan, además es notorio que pese a ser catalogadas como influenciadoras en el rubro de belleza, moda y tendencias, se permiten explorar en temáticas vinculadas con los valores, como un espacio de encuentro más personal con sus seguidores. Así también, y como es el caso de Rosangela, se introduce una referencia a otra red social, en este caso TikTok, como estrategia para movilizar una parte de su audiencia a otra plataforma.

En general, las temáticas presentan una informalidad, pero también momentos de reflexión o inclusión del público digital como es el caso de los sorteos o una coreografía que induzca a la audiencia a replicarla. Se centra los contenidos más importantes a mitad de año, fechas donde no abundan eventos coyunturales dentro del país que puedan modificar el movimiento y acciones ejecutadas por los usuarios.

Tabla 7. Publicaciones con mejor repercusión - Instagramers Colombia

\begin{tabular}{|l|r|c|c|c|c|}
\hline \multicolumn{1}{|c|}{ Instagramer } & Fecha & Hora & Tema & Me Gusta & Comentarios \\
\hline Paula Galindo & $1 / 06 / 19$ & $14: 05$ & Humor de lunes & $125 \mathrm{~K}$ & 259 \\
\hline Daniel Ospina & $1 / 06 / 19$ & $13: 11$ & Llegó Junio & $66 \mathrm{~K}$. & 225 \\
\hline Daniel Ospina & $31 / 05 / 19$ & $15: 25$ & Cambios & $55 \mathrm{~K}$. & 207 \\
\hline Amadorat & $31 / 05 / 19$ & $15: 03$ & Fashion icons & $240 \mathrm{~K}$. & 637 \\
\hline Daniel Ospina & $30 / 05 / 19$ & $19: 27$ & Luz Propia & $130 \mathrm{~K}$. & 400 \\
\hline Daniela Ospina & $30 / 05 / 19$ & $15: 05$ & Agradecimiento & $200 \mathrm{~K}$. & 716 \\
\hline Paula Galindo & $29 / 05 / 19$ & $20: 35$ & Reflexión & $176 \mathrm{~K}$. & 441 \\
\hline Daniela Ospina & $29 / 05 / 19$ & $18: 12$ & Cumpleaños hija & $188 \mathrm{~K}$. & $1.2 \mathrm{~K}$. \\
\hline Cami Zuluaga & $29 / 05 / 19$ & $17: 50$ & Naturaleza & $43 \mathrm{~K}$. & 75 \\
\hline Matu Garcés & $29 / 05 / 19$ & $17: 50$ & Cambios & $67 \mathrm{~K}$. & 134 \\
\hline
\end{tabular}

Fuente: Fan Page Karma (2020). Elaboración propia. 
En este punto y como se expone en la Tabla 7, se suscita una situación similar a Perú. Las publicaciones con mejor repercusión giran en torno al mes de mayo principalmente y dos en junio. A excepción de dos publicaciones, todas se realizan durante la tarde (viernes 4 publicaciones y 2 publicaciones cada día el sábado, domingo y lunes), justificado al ser Instagram una red social que en su mayoría es usada por adolescentes; este espacio de tiempo es propicio para generar una interacción con ellos. Si bien los perfiles mantienen en todo momento una estética que responde al rubro donde pertenecen, es destacable que similar al caso anterior, empiecen a publicar contenido desde nuevas perspectivas, ampliando el bagaje de temáticas que son las que ubican a estas influenciadoras como productoras del material que mayor interacción generó. A diferencia de Perú, en Colombia los cinco perfiles seleccionados, sin excepción, consiguen un lugar dentro de las 10 publicaciones con mejor impacto respecto al movimiento en la red, de igual forma, tanto los "me gusta" como los "comentarios", son proporcionales al número de seguidores que acarrea cada influenciadora.

Como particularidad, solo uno de los temas que mayor cantidad de interacción producen se refiere a la moda y estética, el resto abarca al igual que en Perú, diversidad de enfoques. Se considera que, aunque existe un rubro en particular, se tiene la necesidad de crear contenido alterno también, donde la identidad quede reflejada, pero también se demuestre capacidad de elaboración, producción y difusión de otros temas. La belleza y las tendencias se integran de una forma u otra, pero se van ideando formas de incluirlas sin acentuar directamente en ellas.

\section{Discusión y conclusiones}

Las Instagramers de Perú y Colombia desarrollan una interacción con sus seguidores fundamentada principalmente en los "me gusta" y "comentarios" generados a partir de una publicación. La ejecución de otras reacciones como el compartir los contenidos u otro tipo de reacción, se efectúan en menor medida, aspecto que queda comprobado al relacionar las dos variables referentes a los "me gusta" obtenidos y a la expansión por otras vías de los posteos realizados.

La actividad entre las influenciadoras y sus audiencias presenta algunas variaciones en Perú y Colombia; si bien en el primer país las influenciadoras acumulan millones de participaciones de usuarios, en Colombia los índices se elevan, principalmente en aquellas que se posicionan como líderes. Aunque un hecho que se repite en ambos grupos es la disminución en cuanto a la emisión de texto escrito por el público, un accionar que demuestra una audiencia con una tendencia a la inmediatez y que, sin bien gusta de un contenido, no destina mayor tiempo a opinar en él.

Es indiscutible que el número de seguidores que acumulan determinan de forma directa el nivel de participación que se registra dentro de sus perfiles, sin embargo, el grado de compromiso de las influenciadoras como símbolo de interacción y lealtad de sus audiencias, no presenta una relación directa con cuantos seguidores tiene. En el caso de Colombia y aunque existe un liderazgo prominente, otro perfil adquiere la mejor perspectiva en cuanto compromiso. Este aspecto va más allá de un "me gusta" a un perfil, sino que abarca una interacción constante, confianza y una percepción positiva por parte de las audiencias digitales.

Las cuentas principales de Perú y Colombia empiezan a explorar otros espacios que demuestren no solo lo que venden o promocionan, sino sus criterios personales e incluso, lo que hay detrás de ellas a nivel personal. Es cierto que la belleza, moda y tendencias se convierten en un rubro clave a nivel visual, más aún en una plataforma donde la imagen tiene un peso muy alto y es pieza clave para la posterior distribución de un mensaje, pero demuestran una ampliación de sus horizontes respecto a lo que incluyen, compaginándolo así un rubro considerando un tanto materialista con mensajes centrados en la persona o su bienestar. Esto queda de manifiesto en los posteos que mayor interacción generan durante el 2019, casi en su totalidad vinculados temáticas variadas. 
Es claro que detrás los posteos con mayor repercusión realizados, existe una estrategia respecto al tiempo y fechas en que se lo hace. Tanto en el caso de Perú y Colombia la publicación de contenido se efectúa en la tarde o noche, siempre acercándose a partir del viernes y el fin de semana; únicamente en el caso de Colombia este periodo se extiende a inicio de semana, pero en términos generales se centra en periodo jueves-domingo.

\section{Bibliografía}

Asim, Y., Malik, A., Raza, B. y Shahid, A. (2019). A trust model for analysis of trust, influence and their relationship in social network communities. Telematics and Informatics, 36, 94-116. https://doi.org/10.1016/j.tele.2018.11.008

Arora, A., Bansal, S., Kandpal, C., Aswani, R. y Dwivedi, Y. (2019). Measuring social media influencer index- insights from Facebook, Twitter and Instagram. Journal of Retailing and Consumer Services, Vol. 49, 86-101. https://doi.org/10.1016/j.jretconser.2019.03.012

Baulch, E. y Pramiyanti, A. (2018). Hijabers on Instagram: Using Visual Social Media to Construct the Ideal Muslim Woman. Social Media + Socety, 4(4), 1-15. https://doi.org/10.1177/2056305118800308

Casaló, L., Flavián, C. e Ibáñez-Sánchez, S. (2018). Influencers on Instagram: Antecedents and consequences of opinion leadership. Journal of Business Research, Article in Press, 1-10. https:// doi.org/10.1016/j.jbusres.2018.07.005

Colliander, J. y Marder, B. (2018). 'Snap happy’ brands: Increasing publicity effectiveness through a snapshot aesthetic when marketing a brand on Instagram. Computers in Human Behavior, 78, 34-43. https://doi.org/10.1016/j.chb.2017.09.015

Couture, A. (2020). The looking glass selfie: Instagram use frequency predicts visual attention to high-anxiety body regions in young women. Computers in Human Behavior, 108, 1-12. https:// doi.org/10.1016/j.chb.2020.106329

Despard, E. (2015). Photographic social media, designed landscapes and urban, place-based visibilities: in search of friction. Journal of Aesthetics \& Culture, 7(1), 1-15. https://doi. org/10.3402/jac.v7.28242

Fernández, E. (2016). Juegos olímpicos, televisión y redes sociales. Editorial UOC.

Gómez, E. (2012). De la cultura Kodak a la imagen en red: una etnografia sobre fotografía digital. Editorial UOC.

Martínez-Sanz, R. y González-Fernández, C. (2018). Comunicación de Marca en Instagram, ¿Una Cuestión de Género? El rol del influencer de moda. Masculinities and Social Change,7(3), 230254. https://doi.org/10.17583/MCS.2018.3693

Matley, D. (2018). "Let's see how many of you mother fuckers unfollow me for this": The pragmatic function of the hashtag \#sorrynotsorry in non-apologetic Instagram posts. Journal of Pragmatics, 133, 66-78. https://doi.org/10.1016/j.pragma.2018.06.003

Huang, Y. y Su, S. (2018). Motives for Instagram Use and Topics of Interest among Young Adults. Future Internet, 10(77), 1-12. https://doi.org/10.3390/fi10080077

Hurley, Z. (2019). Imagined Affordances of Instagram and the Fantastical Authenticity of Female Gulf-Arab Social Media Influencers. Social Media + Society, 5(1), 1-16. https://doi. org/10.1177/2056305118819241 
Ichau, E., Frissen, T. y d'Haenens, L. (2019). From \#selfie to \#edgy. Hashtag networks and images associated with the hashtag \#jews on Instagram. Telematics and Informatics, 44, 1-16. https://doi. org/10.1016/j.tele.2019.101275

Jackson, C. y Luchner, A. (2018). Self-presentation mediates the relationship between Selfcriticism and emotional response to Instagram feedback. Personality and Individual Differences, 133, 1-6. https://doi.org/10.1016/j.paid.2017.04.052

Kim, J. y Kim, Y. (2019). Instagram user characteristics and the color of their photos: Colorfulness, color diversity, and color harmony. Information Processing Management, 54, 14941505. https://doi.org/10.1016/j.ipm.2018.10.018

Lario, J.E. (2019). Técnicas de persuasión y comunicación digital. Caligrama.

Larsson, A. (2017). The News User on Social Media. Journalism Studies, 19(15), 2225-2242. https://doi.org/10.1080/1461670X.2017.1332957

Lee, C. y Chau, D. (2018). Language as pride, love, and hate: Archiving emotions through multilingual Instagram hashtags. Discourse, Context \& Media, 22, 21-29. https://doi. org/10.1016/j.dcm.2017.06.002

Longobardi, C., Settani, M., Fabris, M.A. y Marengo, D. (2020). Follow or be followed: Exploring the links between Instagram popularity, social media addiction, cyber victimization, and subjective happiness in Italian adolescents. Children and Youth Service Review, 113, 1-7. https://doi.org/10.1016/j.childyouth.2020.104955

Lowe-Calverley, E., Grieve, R. y Padgett, C. (2020). A risky investment? Examining the outcomes of emotional investment in Instagram. Telematics and Informatics, 49, 1-11. https://doi. org/10.1016/j.tele.2019.101299

Mukherjee, P. y Jansen, B. (2020). Influence of Social Media Attitude in Cross Screen Conversation. Procedia Computer Science, 168, 129-138. https://doi.org/10.1016/j. procs.2020.02.275

Nandagiri, V. y Philip, L. (2018). The impact of influencers from Instagram and YouTube on their followers. International Journal of Multidisciplinary Research and Modern Education, 4 (1), 61-65.

Padrini, P. (2015). Facebook, internet y los medios digitales. Una guía para los padres de familia y los educadores. Editorial San Pablo.

Parmeleee, J. y Roman, N. (2020). Insta-Echoes: Selective Exposure and Selective Avoidance on Instagram. Telematics and Informatics, 52, 1-37. https://doi.org/10.1016/j. tele. 2020.101432

Rietveld, R., Van Dolen, W., Mazloom, M. y Worring, M. (2020). What You Feel, Is What You Like Influence of Message Appeals on Customer Engagement on Instagram. Journal of Interactive Marketing, Vol. 49, 20-53. https://doi.org/10.1016/j.intmar.2019.06.003

Riquelme, H. (2017). Instagram: its influence to psychologically empower women. Information Technology \& People, 31(6), 1113-1134. https://doi.org/10.1108/ITP-03-2017-0079

Stevenson, O., Oluranti, M., Edemode, J. y Anyanwu, B. (2018). Impact of Social Media on the English Language Writing Abilities of Undergraduates in Ekpoma, Nigeria. Gist Education and Learning Research Journal, 17, 59-80. http://dx.doi.org/10.26817/16925777.412 
Tiggemann, M. y Barbato, I. (2018). "You look great!": The effect of viewing appearance-related Instagram comments on women's body image. Body Image, 25, 61-66. https://doi.org/10.1016/j. bodyim.2018.08.009

Venus, S. y Ryu, E. (2020). "I'll buy what she's \#wearing": The roles of envy toward and parasocial interaction with influencers in Instagram celebrity-based brand endorsement and social commerce. Journal of Retailing and Consumer Services, 55, 1-15. https://doi.org/10.1016/j. jretconser.2020.102121

Weismueller, J., Harrigan, P., Wang, S. y Soutar, G. (2020). Influencer endorsements: How advertising disclosure and source credibility affect consumer purchase intention on social media. Australasian Marketing Journal, Article in Press, 1-11. https://doi.org/10.1016/j. ausmj.2020.03.002

Wilkinson, J. (2018). Accessible, Dynamic Web Content Using Instagram. Information Technology and Libraries, 37(1), 19-26. https://doi.org/10.6017/ital.v37i1.10230 